\title{
Tratamiento percutáneo de las oclusiones totales crónicas
}

\section{Parte 1}

\author{
Carlos E. Uribe ${ }^{a, b, c, d, *}$, Mauricio Zúñiga ${ }^{c}$, Jaime Cabrales ${ }^{e}$, Libardo Medina ${ }^{f}$ \\ y Federico Saaibi ${ }^{f}$ \\ a Clínica CardioVID. Medellín, Colombia \\ b Hospital Pablo Tobón Uribe. Medellín, Colombia \\ c Programa de Hemodinamia y Cardiología Intervencionista, Facultad de Medicina, Universidad Pontificia Bolivariana. Medellín, \\ Colombia \\ d Universidad CES. Medellín, Colombia \\ e Hemodinamia y Cardiología intervencionista, Fundación Cardioinfantil-Instituto de Cardiología. Bogotá, Colombia \\ ${ }^{f}$ Medicina Interna y Cardiología, Fundación Cardiovascular de Colombia, Floridablanca, Colombia
}

Recibido el 4 de abril de 2017; aceptado el 10 de noviembre de 2017

Disponible en Internet el 21 de diciembre de 2017

\section{PALABRAS CLAVE}

Enfermedad de las arterias coronarias; Intervención coronaria percutánea; Stents liberadores de fármacos; Oclusión

\section{KEYWORDS}

Coronary artery

disease;

Percutaneous

coronary intervention

\begin{abstract}
Resumen El tratamiento intervencionista de las oclusiones totales crónicas ha sido uno de los procedimientos más complejos para el cardiólogo intervencionista, más aun tratándose de un procedimiento que con frecuencia resulta fallido; sin embargo, en los últimos años ha habido un progreso significativo debido a la creación de técnicas novedosas de disección subintimal y de reentrada, la adopción de la técnica retrógrada y el diseño de flujogramas organizados que permitieron conceptualizar la estrategia de recanalización híbrida para el tratamiento de las oclusiones totales crónicas, a fin de alcanzar tasas de éxito nunca antes vistas. Este artículo constituye una revisión de los tratamientos disponibles para las oclusiones totales crónicas basado en la evidencia, en un esfuerzo para unificar conceptos con base en las estrategias existentes. (c) 2017 Publicado por Elsevier España, S.L.U. en nombre de Sociedad Colombiana de Cardiología y Cirugía Cardiovascular. Este es un artículo Open Access bajo la licencia CC BY-NC-ND (http:// creativecommons.org/licenses/by-nc-nd/4.0/).
\end{abstract}

\section{Percutaneous treatment of chronic total occlusions}

Part 1

\begin{abstract}
The interventionist treatment of chronic total occlusions has been one of the most complex procedures for interventionist cardiologists, especially since it is a procedure which frequently fails. However, in recent years there has been significant progress due to the creation of novel subintimal dissection and re-entry techniques, the adoption of the retrograde technique, and the design of organized flow charts which have allowed the conceptualization of the
\end{abstract}

\footnotetext{
* Autor para correspondencia.

Correo electrónico: uribemd72@hotmail.com (C.E. Uribe).
} 
Drug-eluting stents; Occlusions hybrid recanalization strategy for treating chronic total occlusions, achieving unprecedented success rates. This article reviews the available treatments for chronic total occlusions, based on the evidence, in an effort to unify concepts in terms of existing strategies.

(C) 2017 Published by Elsevier España, S.L.U. on behalf of Sociedad Colombiana de Cardiología y Cirugía Cardiovascular. This is an open access article under the CC BY-NC-ND license (http:// creativecommons.org/licenses/by-nc-nd/4.0/).

\section{Introducción}

El tratamiento de las oclusiones totales crónicas siempre ha sido uno de los procedimientos más complejos y difíciles para los cardiólogos intervencionistas, más aun tratándose de un procedimiento que históricamente ha tenido altas tasas de recanalización no exitosa. Pese a ello, en los últimos años ha habido un progreso considerable dado por la creación de técnicas novedosas de disección subintimal y de reentrada, la adopción de la técnica retrógrada y la creación de flujogramas organizados que permitieron conceptualizar la estrategia de recanalización híbrida para el tratamiento de las oclusiones totales crónicas, hecho que ha permitido alcanzar tasas de recanalización nunca antes vistas. Con estos logros, la técnica híbrida de recanalización de las oclusiones totales crónicas es cada vez más reproducible y está al alcance del operador habitual.

\section{Definición}

Las oclusiones totales crónicas se definen como la presencia de flujo TIMI 0 en el segmento ocluido, con una duración estimada de la oclusión de más de tres meses ${ }^{1,2}$. Arterias con oclusiones entre los treinta días, como aquellas del estudio OAT (Open Artery Trial), no se consideran oclusiones totales crónicas $^{3,4}$.

Se ha clasificado la duración de la oclusión según tres tipos de certeza:

- Cierta: confirmada por angiografía tres meses previos.

- Probable: confirmada por parámetros clínicos, no angiográficos.

- Indeterminada ${ }^{1}$.

\section{Prevalencia}

En un estudio reciente llevado a cabo en Canadá en tres centros de referencia, la prevalencia de oclusiones totales crónicas en 14.439 pacientes, fue del 14,7\%. En pacientes con cirugía de revascularización coronaria fue del $54 \%$ y en aquellos con infarto agudo de miocardio con elevación del segmento ST fue del $10 \%^{2}$.

\section{Selección de los pacientes y beneficios de la revascularización}

El beneficio de la revascularización de las oclusiones totales crónicas es mejorar síntomas, función ventricular y otros desenlaces clínicos evaluados en estudios observacionales y registros. El consenso europeo de expertos en oclusiones totales crónicas de 2012, considera que es apropiado intentar la revascularización de las mismas en pacientes sintomáticos o en aquellos que tengan evidencia objetiva de viabilidad o isquemia mayor del $10 \%{ }^{1}$. En las guías del Colegio Americano de Cardiología se permite la recanalización de una oclusión total crónica en pacientes con angina o evidencia objetiva de isquemia (recomendación clase Ila) ${ }^{5}$. Ante oclusiones totales crónicas el proceso de decisión de llevar al paciente a tratamiento de revascularización debe enfocarse en tres pasos: evaluación de los síntomas, valoración del grado de isquemia y demostración de viabilidad.

Los pacientes con oclusiones totales crónicas a menudo tienen síntomas atípicos, que incluso son más frecuentes que los síntomas referidos en pacientes con angina típica ${ }^{6,7}$. Grantham et al. ${ }^{8}$ demostraron que la recanalización exitosa de una oclusión total crónica se asociaba con mejoría de la angina, de la calidad de vida y de la actividad física solo en los pacientes sintomáticos. Galassi et al. ${ }^{9}$ reportaron un mejor desenlace cardiovascular en pacientes revascularizados con defectos de perfusión extensos que en aquellos que tenían perfusiones normales o ligeramente anormales. Se ha reportado que un porcentaje cercano al $12,5 \%$ de isquemia en perfusión miocárdica con isonitrilos, es un punto de corte óptimo para definir el beneficio que obtendrán los pacientes con una oclusión total crónica. Así mismo, aquellos con perfusiones menores del 6,25\% de isquemia tuvieron empeoramiento de la isquemia post-recanalización, hecho que sugiere que el manejo conservador en casos con índices de isquemia bajos, es una opción apropiada ${ }^{10}$. La viabilidad también es un parámetro importante e independiente de la inducción de isquemia. Esta es particularmente útil en pacientes con disfunción ventricular. En tal sentido, el protocolo mediante resonancia magnética nuclear (RMN) identifica mejor aquellos pacientes con viabilidad, que se benefician de recanalizar la oclusión total crónica ${ }^{11,12}$. La figura 1 muestra el flujograma de selección del paciente para recanalización de oclusiones totales crónicas ${ }^{7}$.

\section{Recomendación del Colegio colombiano de Hemodinamia}

- Se recomienda intentar revascularizar una oclusión total crónica cuando haya angina, viabilidad en pacientes con IAM previo, disfunción ventricular o evidencia objetiva de isquemia definida como una perfusión miocárdica con un porcentaje mayor o igual al $10 \%$.

- En la evaluación de viabilidad en oclusiones crónicas, la RMN o la SPECT, son las alternativas recomendadas. 


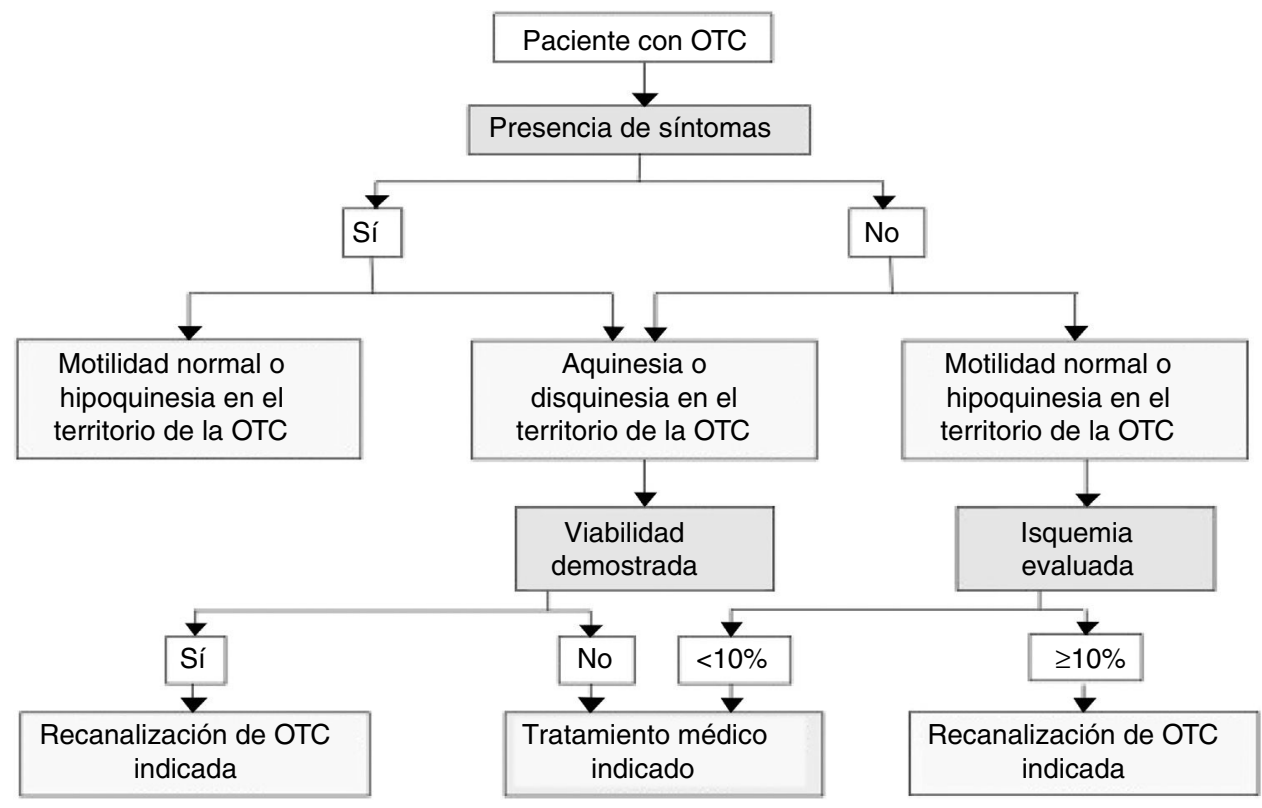

Figura 1 Flujograma para la selección del paciente para recanalización de oclusiones totales crónicas ${ }^{7}$. Reimpreso de: Alfred Galassi et al. Appropriateness of percutaneous revascularization of coronary chronic total occlusions. European Heart. 2015;7:315-24, con permiso de Oxford University Press.

\section{Angina}

La mejoría de la angina es la razón principal para efectuar la recanalización percutánea de una oclusión total crónica. El beneficio en la mejoría de la angina fue demostrado en un metaanálisis reciente de Joyal. Los pacientes que fueron recanalizados exitosamente tuvieron menores cifras de angina recurrente que el grupo que no se recanalizó (OR: $0,45 ; 95 \%$ IC, $0,30-0,67)^{13}$.

\section{Mejoría de la función ventricular}

En pacientes con disfunción ventricular izquierda, la recanalización de las oclusiones totales crónicas demostró mejoría significativa de la función ventricular siempre y cuando el miocardio del área fuera viable y la arteria permaneciera permeable $e^{14,15}$.

\section{Mejoría en la supervivencia}

Estudios observacionales demuestran de manera consecuente una mejoría en la supervivencia en aquellos pacientes en quienes fue exitosa la recanalización de la oclusión total crónica ${ }^{13}$. En un estudio llevado a cabo en un solo centro, el beneficio de la mortalidad fue visto solo en aquellos pacientes en quienes estaba comprometida la arteria descendente anterior ${ }^{16}$.

Una gran limitación del intervencionismo percutáneo de una oclusión total crónica es que a la fecha no existen estudios que hayan aleatorizado el tratamiento médico o la cirugía de revascularización con el tratamiento percutáneo. Hay dos estudios en curso que buscan responder estas preguntas; el primero es el Drug-Eluting Stent Implantation Versus Optimal Medical Treatment in Patients With
Chronic Total Occlusion (DECISION-CTO, NCT01078051), el cual comparará el tratamiento médico contra el tratamiento intervencionista midiendo los desenlaces de muerte, IAM, ataque vascular cerebral y revascularización de cualquier tipo a tres años. El segundo es el European Study on the Utilization of Revascularization versus Optimal Medical Therapy for the Treatment of Chronic Total Coronary Occlusions (EURO-CTO), el cual medirá el punto primario de muerte o IAM no fatal a 36 meses de seguimiento. Se esperan resultados para el año $2018^{3}$.

Un estudio reciente muestra que la mayoría de los pacientes con oclusiones totales crónicas son tratados médicamente (44\%) y solo el $10 \%$ con intervencionismo percutáneo $^{2}$. Es posible que la poca utilización del intervencionismo en estos pacientes obedezca a que sea visto como un procedimiento complejo, con altas tasas de falla. Sin embargo, con las nuevas técnicas, las cifras de éxito del procedimiento han llegado a ser de más del $80 \%$ y en algunas ocasiones hasta de más del $90 \%$ a cargo de operadores expertos en centros de referencia ${ }^{3}$ (fig. 2).

\section{La técnica clásica y el mito del lumen verdadero}

Muchos operadores han hecho esfuerzos increíbles para tratar de permanecer en el lumen verdadero cuando intentan intervenir una oclusión total crónica. La razón que más se esgrime es la seguridad y las complicaciones mínimas, o la creencia de que es más fisiológico o anatómico que la lesión sane o se recupere más rápido luego de implantar un stent.

Para los líderes actuales en el área, el dogma de permanecer en el lumen verdadero es un concepto errado que sólo prolonga el tiempo de intervencionismo y disminuye las tasas de éxito del mismo. Los expertos creen que utilizar 


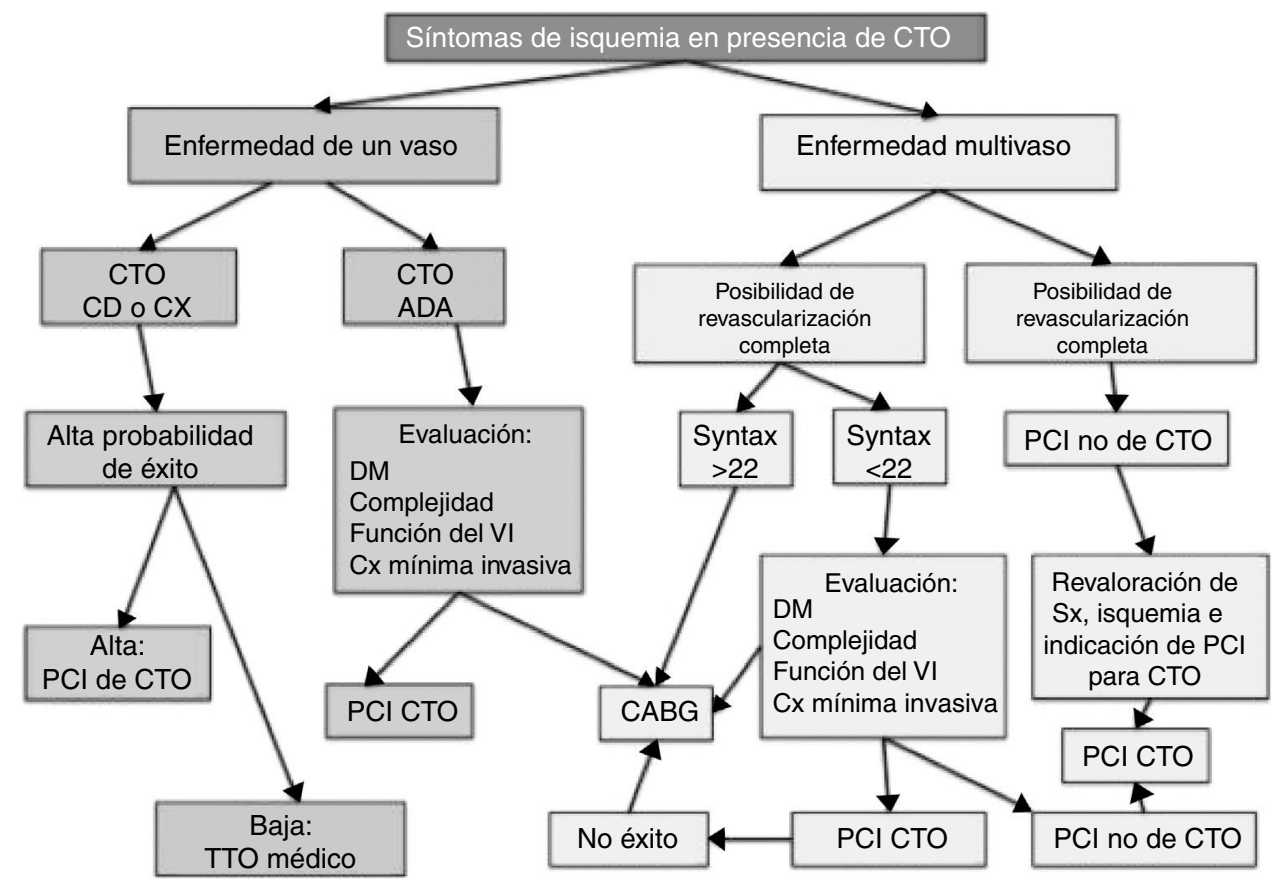

Figura 2 Flujograma de estrategias de manejo para el paciente con oclusión total crónica. CD: arteria coronaria derecha; CX: arteria coronaria circunfleja; ADA: arteria coronaria descendente anterior; $\mathrm{PCl}$ : intervencion coronaria percutánea; TTO: tratamiento; CABG: cirugía de revascularización quirúrgica. Modificada con permiso de: Joyal D, Mansour S. How to Justify CTO Revascularization. In: Rinfret S, editor. Percutaneous intervention for coronary chronic total occlusion: the hybrid approach. Springer International Publishing; 2016. p. 1-11. Con permiso de Springer.

una sola técnica, aumenta el tiempo y las complicaciones, y no resuelve los casos que representan una alta complejidad anatómica. El cardiólogo intervencionista del común tiende a reflejar en su práctica, su zona de confort. El manejo de las oclusiones totales crónicas hoy día, reta los dogmas aprendidos previamente y a menudo es contra-intuitivo para el operador que empieza a tratar las oclusiones totales crónicas. Por ejemplo, tratar de cruzar una guía en loop (técnica del "codo" o knuckle) o cruzar al espacio subintimal con un catéter, es, en opinión de los expertos, más seguro que intentar cruzarlo con guías de punta dura, buscando permanecer en el lumen verdadero. Las técnicas retrógradas son incluso más seguras y rápidas en determinadas anatomías de oclusiones totales crónicas que tratar de hacerlo de manera anterógrada. Otra razón que desvirtúa el mito del lumen verdadero es que se ha demostrado que cuando se utiliza la técnica tradicional para tratar de recanalizar una oclusión total crónica, a menudo se sale a la subíntima y se entra nuevamente al lumen, comportamiento que se ha demostrado en estudios con IVUS en el 30 al 50\% de las veces. También, en cortes histológicos, se ha visto que en las oclusiones totales crónicas hay un remodelamiento del vaso, que no permite la diferenciación completa de las capas; es decir la íntima y lo que tradicionalmente se conoce como lumen verdadero. Es así mismo que al cruzar una oclusión total crónica, no se está en un "lumen verdadero" sino en un vaso que solo tiene dos capas efectivas, la adventicia y la placa ${ }^{17}$. Por lo anterior se debe pensar en una estrategia híbrida al afrontar este tipo de lesiones.

Antiguamente solo se reservaba la técnica retrógrada cuando fallaba la técnica anterógrada; no obstante, a medida que se ha avanzado en el conocimiento y la experiencia de la técnica retrógrada, se considera empezar por esta última en casos donde la oclusión sea difícil o exista tasa alta de falla por vía anterógrada ${ }^{18-20}$.

\section{Planeación del caso}

El análisis cuidadoso de la circulación colateral es clave para planear la estrategia de revascularización. Se debe realizar inyección dual, con catéteres en el ostium izquierdo y derecho, para poder comprender la circulación colateral heterocoronaria a la oclusión y poder determinar si es posible llegar con los dispositivos del lado opuesto. Una proyección oblicua anterior derecha craneal y una oblicua anterior derecha caudal, son claves para visualizar las septales a nivel proximal y distal respectivamente. Lo ideal es un foco pequeño para evitar panear o mover la imagen, pues la relación de las colaterales se precia mejor en imágenes quietas. Los cuadros por segundo podrían ser disminuidos a 7,5 para minimizar la exposición a la radiación. Solo se debe intentar angioplastia ad hoc (coronariografía diagnóstica más angioplastia) si se tiene un tiempo de fluoroscopio de menos de 30 minutos, y el air kerma menor a 3 Gray al momento de intentar recanalizar, teniendo en cuenta que la dosis de 10 Gray se ha considerado el umbral máximo en el cual el operador debe interrumpir el procedimiento ${ }^{21-23}$. De lo contrario, es más razonable y mejor para el paciente hacer el diagnóstico y posteriormente citarle para realizar la angioplastia de la oclusión sin superar dichos umbrales ${ }^{22}$. Por las características de estos procedimientos se deben mantener todas las medidas para reducir la exposición a radiación, como minimizar el tiempo de fluoroscopia, 
reducir las adquisiciones al mínimo, colimar al menor tamaño del campo, minimizar la distancia entre el tubo de rayos $X$ y el paciente, reducir la distancia entre el paciente y el receptor de imagen, utilizar fluoroscopia pulsada con el número de pulsos por segundo más bajo y emplear magnificación solamente cuando sea necesario ${ }^{24}$. Se deberán considerar todas las medidas. Los expertos recomiendan dejar uno o dos días de sala reservados para realizar oclusiones crónicas, pues esto permite concentrar a los pacientes en un solo día, para poder alcanzar una curva de aprendizaje más rápidamente. En algunos casos muy seleccionados de gran complejidad, se recomienda realizar tomografía axial computarizada multicorte coronaria (TAC) para delinear el curso de la arteria, así como para observar el calcio coronario y detalles anatómicos a considerar antes del procedimiento. Así mismo, debe planearse la cantidad de medio de contraste antes del procedimiento. En términos generales, la cantidad máxima de medio de contraste se calcula en $4 \times$ (TFG) o 4 por la tasa de filtración glomerular en $\mathrm{ml} / \mathrm{min}^{1}$.

\section{Recomendación del Colegio colombiano de Hemodinamia}

- La planeación del caso de una oclusión total crónica debe prepararse meticulosamente. No se recomienda la angioplastia "ad hoc" de casos complejos (intervención en el mismo tiempo del diagnóstico).

- Se recomienda concentrar el volumen de pacientes en jornadas o días de angioplastia de oclusiones totales crónicas.

- El procedimiento de revascularización percutánea de una oclusión total crónica debe detenerse ante complicaciones no solucionables, cuando el volumen de contraste alcance 4 x TFG, o cuando la radiación llegue a un límite máximo de 10 Gy de air kerma (K)21-23.

- Se recomienda la inyección con doble catéter (contralateral) antes de intentar la recanalización de una oclusión total crónica.

\section{Acceso}

El acceso es individual según cada grupo y depende de la experiencia de cada operador, incluso puede ser mixto, radial y femoral. Sin embargo, algunos expertos prefieren el acceso femoral ya que permite utilizar diámetros de catéteres mayores de 7 u 8 Fr. La arteria receptora o aquella con la oclusión a tratar, debería ser femoral y tener catéteres de calibre 7 u $8 \mathrm{Fr}$, en tanto que la arteria donante, que es aquella que da las colaterales por donde se pretende cruzar, podría tener un calibre menor $6 \mathrm{Fr}$ y podría ser radial ${ }^{25}$.

\section{Anticoagulación}

Esta se lleva a cabo mediante heparina debido a la seguridad de reversión en caso de perforación. Se evitará la bivalirudina ya que no puede revertirse. No se aconseja el uso de Ilb/Illa por el riesgo de que una perforación mínima pueda convertirse en derrame pericárdico y taponamiento. Se recomienda medir ACT (tiempo de coagulación activado) cada 30 minutos para mantener un ACT de 250 segundos cuando se realiza de forma anterógrada, y de 350 segundos cuando es retrógrada ${ }^{26}$.

\section{Conflictos de interés}

Ninguno.

\section{Bibliografía}

1. Sianos G, Werner GS, Galassi AR, et al., journal of EuroPCR in collaboration with the Working Group on Interventional Cardiology of the European Society of Cardiology. Recanalisation of chronic total coronary occlusions: 2012 consensus document from the EuroCTO club. Eurolntervention:. 2012;8:139-45.

2. Fefer P, Knudtson ML, Cheema AN, et al. Current perspectives on coronary chronic total occlusions: the Canadian Multicenter Chronic Total Occlusions Registry. J Am Coll Cardiol. 2012;59:991-7.

3. Garcia S, Abdullah S, Banerjee S, et al. Chronic total occlusions: patient selection and overview of advanced techniques. Curr Cardiol Rep. 2013;15:334.

4. Hochman JS, Lamas GA, Buller CE, et al. Coronary intervention for persistent occlusion after myocardial infarction. N Engl J Med. 2006;355:2395-407.

5. Levine GN, Bates ER, Blankenship JC, et al. 2011 ACCF/AHA/SCAI Guideline for Percutaneous Coronary Intervention: a report of the American. College of Cardiology Foundation/American Heart Association Task Force on Practice Guidelines and the Society for Cardiovascular Angiography and Interventions. Circulation. 2011;124:e574-651.

6. Galassi AR, Boukhris M, Azzarelli S, et al. Percutaneous coronary interventions for chronic total occlusions: more benefit for the patient or for the interventionist's ego? Can J Cardiol. 2015;31:974-9.

7. Galassi AR, Brilakis ES, Boukhris M, et al. Appropriateness of percutaneous revascularization of coronary chronic total occlusions: an overview. Eur Heart J. 2016;37:2692-700.

8. Grantham JA, Jones PG, Cannon L, Spertus JA. Quantifying the early health status benefits of successful chronic total occlusion recanalization: Results from the FlowCardia's Approach to Chronic Total Occlusion Recanalization (FACTOR) Trial. Circ Cardiovasc Qual Outcomes. 2010;3:284-90.

9. Galassi AR, Werner GS, Tomasello SD, et al. Prognostic value of exercise myocardial scintigraphy in patients with coronary chronic total occlusions. J Interv Cardiol. 2010;23:139-48.

10. Safley DM, Koshy S, Grantham JA, et al. Changes in myocardial ischemic burden following percutaneous coronary intervention of chronic total occlusions. Catheter Cardiovasc Interv. 2011;78:337-43.

11. Kirschbaum SW, Rossi A, Boersma E, et al. Combining magnetic resonance viability variables better predicts improvement of myocardial function prior to percutaneous coronary intervention. Int J Cardiol. 2012;159:192-7.

12. Gerber BL, Rousseau MF, Ahn SA, et al. Prognostic value of myocardial viability by delayed-enhanced magnetic resonance in patients with coronary artery disease and low ejection fraction: impact of revascularization therapy. J Am Coll Cardiol. 2012;59:825-35.

13. Joyal D, Afilalo J, Rinfret S. Effectiveness of recanalization of chronic total occlusions: a systematic review and meta-analysis. Am Heart J. 2010;160:179-87.

14. Baks T, van Geuns RJ, Duncker DJ, et al. Prediction of left ventricular function after drug-eluting stent implantation for chronic total coronary occlusions. J Am Coll Cardiol. 2006;47:721-5.

15. Kirschbaum SW, Baks T, Van den Ent M, et al. Evaluation of left ventricular function three years after percutaneous 
recanalization of chronic total coronary occlusions. Am J Cardiol. 2008;101:179-85.

16. Safley DM, House JA, Marso SP, et al. Improvement in survival following successful percutaneous coronary intervention of coronary chronic total occlusions: variability by target vessel. JACC Cardiovasc Interv. 2008;1:295-302.

17. Sumitsuji S, Inoue K, Ochiai M, et al. Fundamental wire technique and current standard strategy of percutaneous intervention for chronic total occlusion with histopathological insights. JACC Cardiovasc Interv. 2011;4:941-51.

18. Grantham JA, Marso SP, Spertus J, et al. Chronic total occlusion angioplasty in the United States. JACC Cardiovasc Interv. 2009;2:479-86.

19. Ng R, Hui PY, Beyer A, Ren X, Ochiai M. Successful retrograde recanalization of a left anterior descending artery chronic total occlusion through a previously placed left anterior descendingto-diagonal artery stent. J Invasive Cardiol. 2010;22: E16-8.

20. Tsujita K, Maehara A, Mintz GS, et al. Intravascular ultrasound comparison of the retrograde versus anterograde approach to percutaneous intervention for chronic total coronary occlusions. JACC Cardiovasc Interv. 2009;2:846-54.

21. Pavlidis AN, Jones DA, Sirker A, et al. Reducing radiation in chronic total occlusion percutaneous coronary interventions. Curr Cardiol Rev. 2016;12:12-7.

22. Brilakis ES, Grantham JA, Thompson CA, et al. The retrograde approach to coronary artery chronic total occlusions: a practical approach. Catheter Cardiovasc Interv. 2012;79:3-19.

23. Brilakis ES, Grantham JA, Rinfret S, et al. A percutaneous treatment algorithm for crossing coronary chronic total occlusions. JACC Cardiovasc Interv. 2012;5:367-79.

24. Azpiri-Lopez JR, Assad-Morell JL, Gonzalez-Gonzalez JG, et al. Effect of physician training on the $\mathrm{X}$-ray dose delivered during coronary angioplasty. The Journal of invasive cardiology. 2013;25:109-13.

25. Alaswad K. Toolbox and inventory requirements for chronic total occlusion percutaneous coronary interventions. Interventional Cardiology Clinics. 2012;1:281-97.

26. Lembo NJ, Karmpaliotis D, Kandzari DE. CTO PCI Procedural Planning. Interventional Cardiology Clinics. 2012;1:299-308. 